\title{
Volumetric accuracy of different imaging modalities in acute intracerebral hemorrhage
}

\author{
Frieder Schlunk ${ }^{1,2^{*}}$, Johannes Kuthe ${ }^{1}$, Peter Harmel ${ }^{3}$, Heinrich Audebert ${ }^{3}$, Uta Hanning ${ }^{4}$, Georg Bohner ${ }^{1}$,
} Michael Scheel ${ }^{1}$, Justus Kleine ${ }^{1}$ and Jawed Nawabi ${ }^{2,5}$

\begin{abstract}
Background: Follow-up imaging in intracerebral hemorrhage is not standardized and radiologists rely on different imaging modalities to determine hematoma growth. This study assesses the volumetric accuracy of different imaging modalities (MRI, CT angiography, postcontrast CT) to measure hematoma size.

Methods: 28 patients with acute spontaneous intracerebral hemorrhage referred to a tertiary stroke center were retrospectively included between 2018 and 2019. Inclusion criteria were (1) spontaneous intracerebral hemorrhage (supra- or infratentorial), (2) noncontrast CT imaging performed on admission, (3) follow-up imaging (CT angiography, postcontrast $\mathrm{CT}, \mathrm{MRI}$ ), and (4) absence of hematoma expansion confirmed by a third cranial image within 6 days. Two independent raters manually measured hematoma volume by drawing a region of interest on axial slices of admission noncontrast CT scans as well as on follow-up imaging (CT angiography, postcontrast CT, MRI) using a semi-automated segmentation tool (Visage image viewer; version 7.1.10). Results were compared using Bland-Altman plots.
\end{abstract}

Results: Mean admission hematoma volume was $18.79 \pm 19.86 \mathrm{cc}$. All interrater and intrarater intraclass correlation coefficients were excellent (1; IQR 0.98-1.00). In comparison to hematoma volume on admission noncontrast CT volumetric measurements were most accurate in patients who received postcontrast CT (bias of $-2.47 \%$, SD 4.67: $n=10$ ), while CT angiography often underestimated hemorrhage volumes (bias of 31.91\%, SD 45.54; $n=20$ ). In MRI sequences intracerebral hemorrhage volumes were overestimated in T2* (bias of $-64.37 \%, S D 21.65 ; n=10$ ). FLAIR (bias of $6.05 \%$, SD 35.45; $n=13$ ) and DWI (bias of- $14.6 \%, S D 31.93 ; n=12$ ) over- and underestimated hemorrhagic volumes.

Conclusions: Volumetric measurements were most accurate in postcontrast CT while CT angiography and MRI sequences often substantially over- or underestimated hemorrhage volumes.

Keywords: Acute stroke, Imaging, Intracerebral hemorrhage, Neurocritical care, Neuroradiology

\section{Background}

Intracerebral hemorrhage (ICH) is a deadly subtype of stroke and improvement in clinical management is urgently needed. Approximately one third of patients suffer from hematoma expansion after hospital arrival

\footnotetext{
*Correspondence: frieder.schlunk@charite.de

1 Department of Neuroradiology, Charité - Universitätsmedizin Berlin, Corporate Member of Freie Universität Berlin, Humboldt-Universität Zu Berlin, and Berlin Institute of Health, Charitéplatz 1, 10117 Berlin, Germany Full list of author information is available at the end of the article
}

constituting a target for treatment and warranting prompt diagnosis [1]. In the acute setting, non-contrast computed tomography (NCCT) is widely used for initial diagnosis, however, time point and modality of follow-up imaging are typically not standardized [2]. In the clinical course often contrast-enhanced CT scans such as CT-angiography (CTA) or postcontrast CT and MRI are performed to evaluate a secondary underlying cause for $\mathrm{ICH}$, such as cerebral amyloid angiopathy (CAA), neoplastic disease, vasculitis, cavernoma, or other vascular 
abnormalities [3]. Thus, neuroradiologists rely on a different imaging modality for hematoma measurement with intermodal comparison to NCCT admission imaging [4]. However, the accuracy of such intermodal hematoma measurement is not known.

In the hyperacute state, the freshly extravasated blood has similar density as the intravascular blood (30-60 Hounsfield Units, HU), which will soon increase to $80 \mathrm{HU}$ as coagulation proceeds. The high radiodensity allows for an excellent detection rate of intracranial blood with NCCT [5]. CTA has become an integral part of the diagnostic workup because of its high sensitivity for vascular pathologies. In addition, the CTA spot sign, caused by an active extravasation of contrast agent, has been shown to be an indicator of hematoma expansion [6]. CTA is often followed by postcontrast CT to address the presence of underlying neoplastic disease [2].

MRI has been described as accurate as CT in diagnosing the presence of ICH [5]. Furthermore, MRI is the imaging modality of choice to identify CAA or an underlying tumor [7]. However, the MRI appearance of $\mathrm{ICH}$ varies in dependence of applied technique, field strength, and the magnetic properties of the hematoma, which change over time [8].

In this study, we retrospectively assessed the volumetric accuracy of different modalities (MRI, CTA, postcontrast $\mathrm{CT}$ ) to measure hematoma size, in comparison to non-contrast $\mathrm{CT}$ in the first 6 days after $\mathrm{ICH}$.

\section{Methods}

\section{Study population}

The data that support the findings of this study are available from the corresponding author upon reasonable request.

We reviewed clinical data collected as part of the prospectively maintained stroke database of our institution (Charité Universitätsmedizin Berlin). It was searched for all patients with the diagnosis of ICH (time span January 2018 and July 2019). Patients $>18$ years were included if they had a diagnosis of spontaneous intracerebral hemorrhage (supra- or infratentorial) and had received initial NCCT, followed by CTA, postcontrast CT or cranial MRI within the following 6 days, when ICH is still in its subacute stage [9]. To allow adequate comparison of followup imaging to the initial NCCT, a third cranial image was required to exclude patients who experienced interim hematoma growth. Patients referred to our institution from external hospitals were included if initial NCCT was available.

Diagnosis of ICH was established by admission NCCT. On MRI hemorrhage was identified as a characteristic area of hyperintensity or signal loss, respectively, depending on the applied sequence.
Table 1 Comparison of baseline demographic, clinical and radiological characteristics in patients with acute intracerebral hemorrhage $(\mathrm{ICH})$

\begin{tabular}{lc}
\hline Baseline characteristics & $\begin{array}{l}\text { Patients with } \\
\text { acute ICH } \\
(\mathbf{n}=\mathbf{2 8})\end{array}$ \\
\hline Clinical parameters & \\
Age [years], median (IQR) & $76.5(66.5-80.5)$ \\
Female, $\mathrm{n}(\%)$ & $15(53.6)$ \\
Hypertension, $\mathrm{n}(\%)$ & $20(71.4)$ \\
Diabetes mellitus, $\mathrm{n}(\%)$ & $3(10.7)$ \\
GCS score, median (IQR) & $15(13-15)$ \\
Anticoagulation treatment, $\mathrm{n}(\%)$ & $4(14.3)$ \\
Antiplatelet treatment, $\mathrm{n}(\%)$ & $8(28.6)$ \\
NIHHS admission, median (IQR) & $5(2-11)$ \\
NIHSS discharge, median (IQR) & $1(0-8)$ \\
mRS discharge, median (IQR) & $4(1-4)$ \\
CT parameters & \\
Bleeding location, $\mathrm{n}$ (\%) & \\
Lobar & $15(53.6)$ \\
Basal Ganglia & $6(21.4)$ \\
Thalamus & $4(14.3)$ \\
Brainstem and Pons & $0(0.0)$ \\
Cerebellar & $3(10.7)$ \\
Intraventricular hemorrhage, $\mathrm{n}(\%)$ & $8(28.6)$ \\
Midlineshift [mm], median (IQR) & $0.0(0.0-3.0)$ \\
\hline GCS Glasgow Coma Sca $m$ (IS & \\
\hline
\end{tabular}

GCS Glasgow Coma Scale, mRS modified Rankin Scale, NIHSS National Institutes of Health Stroke Scale

The population included patients with anticoagulant treatment, but excluded patients with head trauma, brain tumor, vascular malformation, primary intraventricular hemorrhage, or secondary ICH from hemorrhagic transformation of ischemic infarction. Patients, subjected to surgical procedures (e.g. hematoma evacuation or decompressive craniectomy) were also excluded.

The retrospective study was approved by the responsible ethics committee (Ethik-Komission der Charité Berlin; identification number EA4/011/20). Informed consent of individual patients was waived for this retrospective study with anonymized data according to pertinent institutional guidelines. The study was conducted in accordance with the declaration of Helsinki.

\section{Image acquisitions}

CT scans at the Charité University Hospital were performed on a 80 or 320 slice scanner (Toshiba Aquilion Prime) with the following imaging parameters: NCCT and postcontrast $\mathrm{CT}$ with incremental acquisition at $120 \mathrm{kV}, 280 \mathrm{~mA}, 1.0 \mathrm{~mm}$ slice reconstruction; postcontrast $\mathrm{CT}$ images were acquired $3 \mathrm{~min}$ after contrast agent application; CTA: dose-modulated (100-450 mA) spiral 

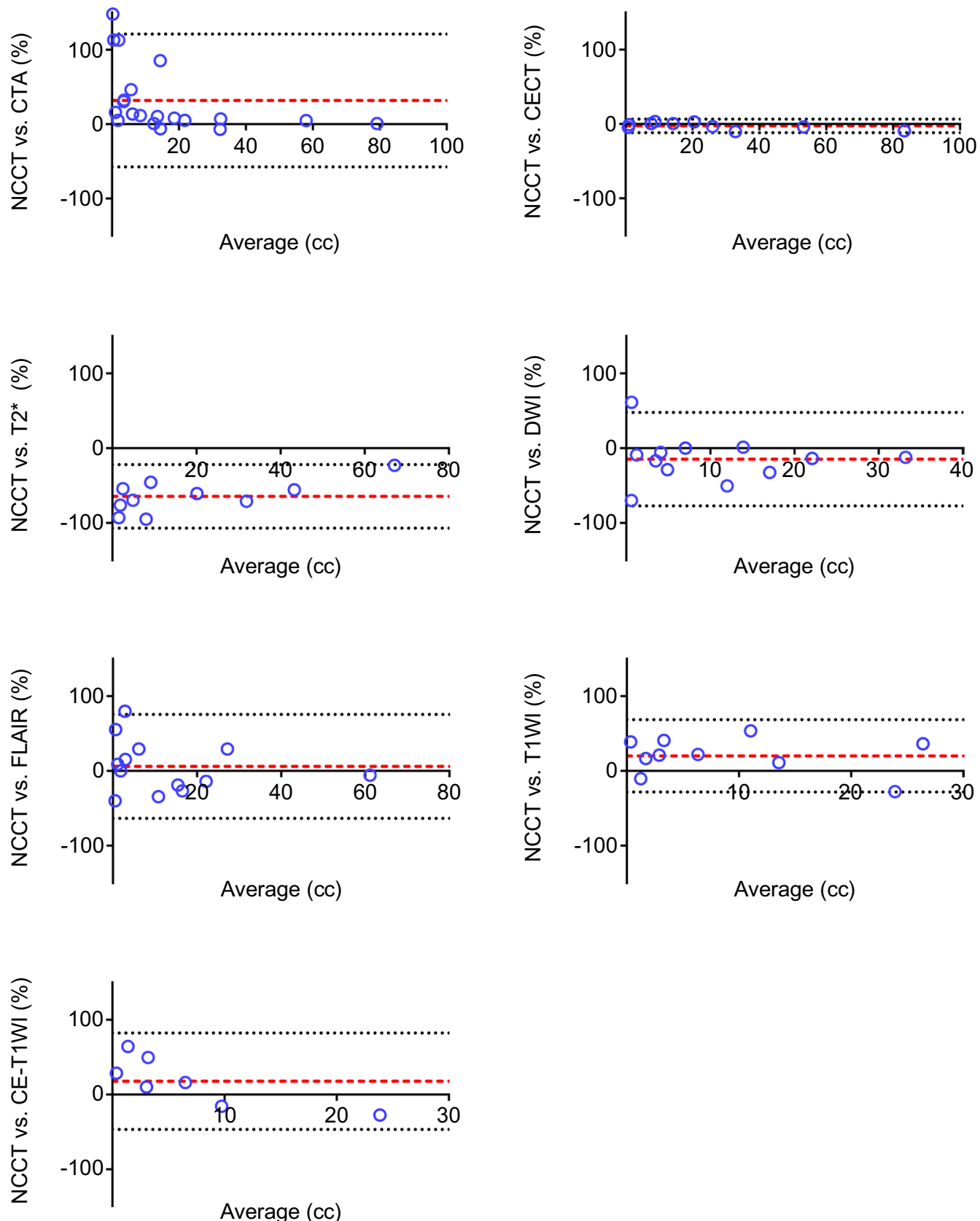

Fig. 1 Bland-Altman plots of non-contrast Computed Tomography (NCCT) versus CT-Angiography (CTA) and postcontrast CT (CECT; upper row) and NCCT versus different MRI sequences (second and third row). T2* indicates T2*-Gradient Echo, DWI indicates Diffusion weighted imaging, FLAIR indicates Fluid attenuated Inversion Recovery Sequences, T1WI and CE-T1WI indicate T1w Spin-Echo pre and post gadolinium intravenous administration respectively. Dashed red lines represent the bias (mean of the difference between measurements). Dotted gray lines represent the limits of agreement (mean $\pm 1.96 \mathrm{SD})$

CT acquisition at $100-120 \mathrm{kV}, 0.5-\mathrm{mm}$ collimation, 0.8 pitch; H2Of soft kernel reconstruction, with $1.0 \mathrm{~mm}$ standard slices and $5 \mathrm{~mm}$ maximum intensity projection (MIP) images with $1 \mathrm{~mm}$ increment. $60 \mathrm{~mL}$ highly iodinated contrast medium and $30 \mathrm{~mL} \mathrm{NaCl}$ flush at $4 \mathrm{~mL} / \mathrm{s}$; scan triggered by bolus tracking at within the ascending aorta with a 6 s delay.

MR scanners used in this study were newest generation 1.5-T and $3 \mathrm{~T}$ (Siemens Medical Solutions, Erlangen, Germany) respectively. A standard head coil was used. 
The MRI protocol included T2\%-Gradient Echo (slice thickness $=2-3 \mathrm{~mm}$ ), Diffusion weighted imaging (DWI, slice thickness $=2-3 \mathrm{~mm}$ ), Fluid attenuated Inversion Recovery Sequences (FLAIR, slice thickness $=2-3 \mathrm{~mm}$ ) and T1w Spin-Echo (slice thickness $=2-3 \mathrm{~mm}$ ) pre and post gadolinium intravenous administration.

\section{Image analysis}

Two raters, experienced in stroke imaging (F.S., J.K.) independently reviewed images in a random order, blinded to all demographic and outcome data. Readers were not involved in the clinical assessment or care of the enrolled patients. Images were re-randomized and presented again to one rater (F.S.) one month later for a second reading. First, the location of the hematoma and presence of intraventricular hemorrhage were assessed and documented by reviewing admission CT images. Also the hemorrhage locations were classified as deep (basal ganglia and thalamic), lobar, brain stem, and cerebellum on admission CT images. Thereafter, regions of interest (ROIs) were delineated on axial slices of images in the different imaging modalities (admission and follow-up) using a semi-automated segmentation tool provided in the Visage image viewer (version 7.1.10). On CT images measurements were completed between 20 and 80 Hounsfield units (HU) to exclude voxels that likely belong to cerebrospinal fluid or calcification. Discrepancies were settled by joint discussion of the 2 readers.

\section{Statistical analysis}

Clinical data from our stroke database and results from manual segmentation were statistically analyzed as following: For baseline data, mean and standard deviations (SD) were used for normally distributed, and median and range for non-normally distributed data, respectively. To test for normality the D'Agostino-Pearson test was used. Categorical variables are reported as counts and percentages. $P$ values less than 0.05 were considered significant. Intrarater and interrater reliability were measured with intraclass correlation coefficients (ICC) using the statistical software package SPSS version $25^{\circledR}$ (IBM Corporation, Armonk NY). ICC was interpreted as following: Moderate agreement (0.41-0.60), substantial agreement (0.61-0.80), and almost perfect (excellent) (0.81-1.00)
[10]. GraphPad Prism 7 version 7.00 was used for BlandAltman plots to determine the volumetric accuracy of the above mentioned imaging modalities in comparison to initial NCCT.

\section{Results}

From January 2018 to June 2019, 286 patients were identified in our database with the diagnosis of primary acute $\mathrm{ICH}$. Of those, 28 consecutive patients were included, who received initial NCCT as well as at least two followup imaging studies, and did not show interim hematoma enlargement. Patients had a median age of 76.5 (66.5$80.5), 15$ (53.6\%) were female.

Of the 28 patients with initial NCCT 20 received CTA as a follow up, 10 postcontrast CT, and 14 MRI. Duration from initial NCCT to first follow-up imaging was a median of $16 \mathrm{~h}$ (IQR 1-28 h); to second follow-up imaging a median of $59 \mathrm{~h}$ (IQR 23-98 h). Further baseline demographics and clinical characteristics are shown in Table 1.

ICH was deep hemispheric in 10 (35.7\%), lobar in 15 (53.6\%), and infratentorial in 3 subjects (10.7\%). Mean hematoma volume at admission was $18.79 \pm 19.86 \mathrm{cc}$.

All interrater and intrarater ICCs were excellent (1; IQR 0.98-1.00) for both CT and MRI scans.

In comparison to the initial NCCT, volumetric measurements were most accurate in patients who received postcontrast CT (bias of $-2.47 \%$, SD 4.67: $n=10$ ), while CTA often underestimated hemorrhage volumes, especially in bleeds $<20 \mathrm{cc}$ (bias of $31.91 \%, \mathrm{SD} 45.54 ; \mathrm{n}=20$; Bland-Altman plots in Figs. 1, 2a, b; Additional file 1: Table S1).

In MRI sequences ICH volumes were generally overestimated in T2* (bias of $-64.37 \%$, SD 21.65; $\mathrm{n}=10$ ). T1WI (bias of $20.23 \%$, SD 24.65; $\mathrm{n}=10$ ) and contrast enhanced T1WI (bias of $17.96 \%$, SD 32.9; $\mathrm{n}=7$ ) both showed a trend to measure hematoma volumes smaller compared to NCCT. The most accurate MRI sequences in terms of comparability to the initial NCCT were DWI and FLAIR, which showed higher relative deviations in both directions however (bias of-14.6\%, SD 31.93; $\mathrm{n}=12$; bias of $6.05 \%$, SD 35.45; $n=13$ respectively; Figs. 1, 2c; Additional file 1: Table S1).

\footnotetext{
(See figure on next page.)

Fig. 2 a Comparison of the same patient with ICH in the right occipital lobe (arrowhead) using different imaging modalities. The borders of the hematoma can be seen with clear contrast on admission NCCT (upper row) and follow-up postcontrast CT (lower row). On CTA (middle row) borders of the hematoma are difficult to distinguish from surrounding parenchyma. $\mathbf{b}$ In this example of a patient with right temporoparietal ICH (arrowhead), hematoma appears to be smaller on follow-up CTA (lower row) compared to admission NCCT (upper row). c Right thalamic ICH on NCCT, FLAIR, DWI and T2* (from left to right). On MRI sequences hemorrhage are more heterogeneous and borders are not as easily to distinguish from the surrounding tissue. Hemorrhage appears to be bigger on T2*
} 

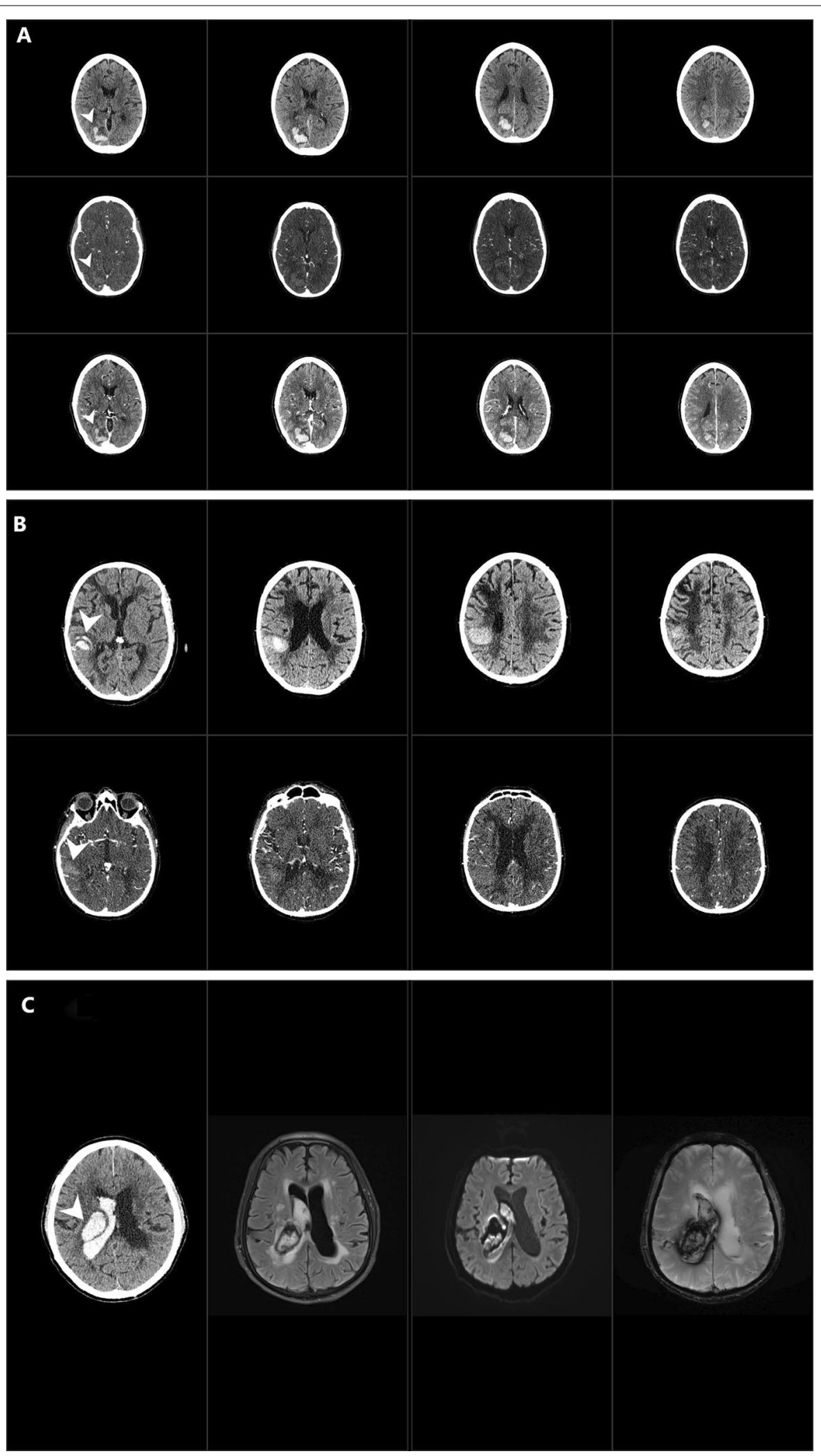

Fig. 2 (See legend on previous page.) 


\section{Discussion}

In this retrospective study we investigated the accuracy of volumetric measurements of $\mathrm{ICH}$ volumes in different imaging modalities. To our knowledge, this is the first study analyzing the accuracy of volumetric measurements of ICH in NCCT in comparison to CTA, or postcontrast CT. We found an excellent correlation of hemorrhage volume measured on postcontrast $\mathrm{CT}$ in comparison to admission NCCT, while hematoma size on CTA was underestimated in some cases. In MRI sequences (DWI, T2*, FLAIR, T1WI with and without $\mathrm{CE})$ hemorrhage size was measured with a substantial bias.

While NCCT has been the gold standard for the diagnosis and for follow-up of ICH for many years, MRI has gained utility in the acute setting, due to its excellent detection rate for ischemic and hemorrhagic stroke. As in a previous study from 1999, T2*-MRI clearly overestimated hematoma size [11]. While that study concluded that DWI and FLAIR were the most accurate MRI techniques, with a good correspondence to NCCT, we also found that DWI and FLAIR were the least inaccurate MRI-sequences, but tended to over- and underestimate, respectively, smaller hematoma volumes $<20 \mathrm{cc}$. That readers were blinded to initial NCCT results and clinical data in our study (which was not explicitly mentioned in that previous study), the use of current generation MRI and CT hardware, and the fact that we explicitly excluded patients with interim hematoma growth might have contributed to this difference.

The main finding is that postcontrast $\mathrm{CT}$ is excellently suited to compare hematoma size with NCCT. This finding could have direct clinical implications, proposing postcontrast $\mathrm{CT}$ in patients with high-risk of clinical worsening as the preferred tool (a) to check for further hematoma expansion, (b) to assess venous pooling as a surrogate for active bleeding, and (c) to identify malignancies as underlying pathology [12]. A protocol using a slightly delayed postcontrast CT after initial NCCT would also be a suitable tool in clinical trials targeting hematoma expansion, saving costs and sparing patients substantial radiation dose for an additional NCCT-scan, which would otherwise be the most obvious alternative for precise assessment of hematoma expansion. By contrast, radiologists should be careful to diagnose hematoma progression or regression on CTA, if no additional confirmatory imaging modality is available.

Several strengths of the present study contribute to the robustness of our findings. First, newest generation scanners were used in our study, and second, all images were examined by experienced radiologists blinded to initial NCCT and relevant clinical data. Limitations are the retrospective design, and the moderate sample size. While similar case numbers can be found for comparable studies in the literature we understand our work as a pilot study.
Careful interpretation is therefore warranted and prospective validation of the results in a larger patient cohort will be needed in the future $[11,13,14]$. While our dataset comprised both small and large hemorrhages, clinically severe cases of ICH might be underrepresented in our dataset (GCS 15 (13-15; Additional file 2: Table S2)). Furthermore we did not include data on intraventricular hemorrhage, which we plan to investigate in future work.

\section{Conclusions}

Postcontrast CT offers excellent interrater and intrarater reliabilities to measure hematoma size in follow-up imaging after acute $\mathrm{ICH}$ and offers a rationale for a standardized follow-up imaging protocol. Hematomas on CTA should not be compared with initial NCCT since it regularly underestimates hematoma volumes. While MRI has excellent sensitivity for the qualitative detection of $\mathrm{ICH}$, quantitative measurement of hematoma size may not be sufficiently accurate for a precise comparison with $\mathrm{NCCT}$ acquired on admission.

\section{Abbreviations}

MRI: Magnetic resonance imaging; CTA: Computed tomography angiography; CT: Computed tomography; ICH: Intracerebral hemorrhage; ICC: Intraclass correlation coefficient; IQR: Interquartile range; NCCT: Non-contrast computed tomography; CAA: Cerebral amyloid angiopathy; kV: Kilovolt; mA: Milliampere; MIP: Maximum intensity projection; NaCl: Sodium chloride; T: Tesla; DWI: Diffusion weighted imaging; FLAIR: Fluid attenuated inversion recovery; T1WI:T1 weighted image; ROI: Region of interest; HU: Hounsfield units; SD: Standard deviation; CE: Contrast Enhanced; GCS: Glasgow coma score.

\section{Supplementary Information}

The online version contains supplementary material available at https://doi. org/10.1186/s12880-022-00735-3.

Additional file 1: Table S1. Absolute ICH volumes in non-contrast Computed Tomography (NCCT; left side) and follow-up imaging modality (right side).

Additional file 2: Table S2. Comparison of baseline demographic, clinical and radiological characteristics in patients with acute intracerebral hemorrhage (ICH; study cohort vs. comparison group). EVD indicates extraventricular drainage; GCS, Glasgow Coma Scale; mRS modified Rankin Scale; NIHSS National Institutes of Health Stroke Scale.

\section{Acknowledgements}

Frieder Schlunk is participant in the BlH-Charité Clinician Scientist Program funded by the Charité -Universitätsmedizin Berlin and the Berlin Institute of Health. Jawed Nawabi is grateful for being part of the BIH Charité - Digital Clinician Scientist Program funded by Charité-Universitaetsmedizin Berlin, the Berlin Institute of Health and the German Research Foundation (DFG, Deutsche Forschungsgemeinschaft).

\section{Authors' contributions}

FS: Conception and design, acquisition of data, analysis and interpretation of data, drafting the article, final approval of the version to be published; JJK: Acquisition of data, revising the manuscript critically, final approval of the manuscript; $\mathrm{PH}$ : Analysis and interpretation of data, revising the manuscript critically, final approval of the manuscript; $\mathrm{HA}$ : Analysis and interpretation of data, revising the manuscript critically, final approval of the manuscript; $U H$ : 
Analysis and interpretation of data, revising the manuscript critically, final approval of the manuscript; GB: Analysis and interpretation of data, revising the manuscript critically, final approval of the manuscript; MS: Substantial contributions to conception and design, revising the manuscript critically, final approval of the manuscript; JFK: Analysis and interpretation of data, revising the manuscript critically, final approval of the manuscript; JN: Substantial contributions to conception and design, analysis and interpretation of data, revising the manuscript critically, final approval of the manuscript. All authors read and approved the final manuscript.

\section{Funding}

Open Access funding enabled and organized by Projekt DEAL.

\section{Availability of data and materials}

The data that support the findings of this study are available from the corresponding author upon reasonable request.

\section{Declarations}

\section{Ethics approval and consent to participate}

The retrospective study was approved by the responsible ethics committee (Ethik-Komission der Charité Berlin; Identification Number EA4/011/20). Informed consent of individual patients was waived for this retrospective study with anonymized data according to pertinent institutional guidelines.

\section{Consent for publication}

Not applicable.

\section{Competing interests}

Heinrich Audebert reports funding from the German Research Foundation (DFG), the federal Ministry of Education and Research (BMBF), Berlin Innovation Funds, Pfizer and German Stroke Foundation. He reports speaker fees and consultancy honoraria during the conduct of the study from Bayer Vital, Boehringer Ingelheim Pharma, Bristol-Myers Squibb, Novo Nordisk, Pfizer, and Takeda.

\section{Author details}

${ }^{1}$ Department of Neuroradiology, Charité - Universitätsmedizin Berlin, Corporate Member of Freie Universität Berlin, Humboldt-Universität Zu Berlin, and Berlin Institute of Health, Charitéplatz 1, 10117 Berlin, Germany. ${ }^{2}$ Berlin Institute of Health $(\mathrm{BIH})$, BIH Biomedical Innovation Academy, Berlin, Germany. ${ }^{3}$ Department of Neurology, Charité - Universitätsmedizin Berlin, Campus Mitte, Humboldt-Universität Zu Berlin, Freie Universität Berlin, Charitéplatz 1, 10117 Berlin, Germany. ${ }^{4}$ Department of Diagnostic and Interventional Neuroradiology, University Medical Center Hamburg Eppendorf, Hamburg, Germany. ${ }^{5}$ Department of Radiology (CCM), Charité - Universitätsmedizin Berlin, Campus Mitte, Humboldt-Universität Zu Berlin, Freie Universität Berlin, Charitéplatz 1, 10117 Berlin, Germany.

Received: 23 February 2021 Accepted: 9 January 2022

Published online: 15 January 2022

\section{References}

1. Morotti A, Goldstein JN. Diagnosis and management of acute intracerebral hemorrhage. Emerg Med Clin N Am. 2016:34:883-99.

2. Hemphill JC 3rd, Greenberg SM, Anderson CS, Becker K, Bendok BR, Cushman M, et al. Guidelines for the management of spontaneous intracerebral hemorrhage: a guideline for healthcare professionals from the American Heart Association/American Stroke Association. Stroke. 2015:46:2032-60.

3. Macellari F, Paciaroni M, Agnelli G, Caso V. Neuroimaging in intracerebral hemorrhage. Stroke. 2014;45:903-8.

4. Heit JJ, IV M, Wintermark M. Imaging of intracranial hemorrhage. J Stroke. 2017:19:11-27.

5. Romero JM, Rosand J. Hemorrhagic cerebrovascular disease. Handb Clin Neurol. 2016:135:351-64.

6. Morotti A, Boulouis G, Dowlatshahi D, Li Q, Barras CD, Delcourt C, et al. Standards for detecting, interpreting, and reporting noncontrast computed tomographic markers of intracerebral hemorrhage expansion. Ann Neurol. 2019;86:480-92.

7. Wijman CA, Venkatasubramanian C, Bruins S, Fischbein N, Schwartz N. Utility of early MRI in the diagnosis and management of acute spontaneous intracerebral hemorrhage. Cerebrovasc Dis. 2010;30:456-63.

8. Dainer HM, Smirniotopoulos JG. Neuroimaging of hemorrhage and vascular malformations. Semin Neurol. 2008;28:533-47.

9. Aguilar MI, Brott TG. Update in intracerebral haemorrhage. Neurohospitalist. 2011;1:148-59.

10. Landis JR, Koch GG. The measurement of observer agreement for categorical data. Biometrics. 1977;33:159-74.

11. Schellinger PD, Jansen $O$, Fiebach JB, Hacke W, Sartor K. A standardized $\mathrm{MRI}$ stroke protocol: comparison with CT in hyperacute intracerebral hemorrhage. Stroke. 1999;30:765-8.

12. Chakraborty S, Alhazzaa M, Wasserman JK, Sun YY, Stotts G, Hogan MJ, et al. Dynamic characterization of the CT angiographic "spot sign." PLoS ONE. 2014;9:e90431.

13. Linfante I, Llinas $\mathrm{RH}$, Caplan LR, Warach S. MRI features of intracerebral hemorrhage within 2 hours from symptom onset. Stroke. 1999;30:2263-7.

14. Patel A, Schreuder F, Klijn CJM, Prokop M, Ginneken BV, Marquering HA, et al. Intracerebral haemorrhage segmentation in non-contrast CT. Sci Rep. 2019;9:17858.

\section{Publisher's Note}

Springer Nature remains neutral with regard to jurisdictional claims in published maps and institutional affiliations.
Ready to submit your research? Choose BMC and benefit from:

- fast, convenient online submission

- thorough peer review by experienced researchers in your field

- rapid publication on acceptance

- support for research data, including large and complex data types

- gold Open Access which fosters wider collaboration and increased citations

- maximum visibility for your research: over $100 \mathrm{M}$ website views per year

At BMC, research is always in progress.

Learn more biomedcentral.com/submissions 